Ciencia y Educación, Vol. 4, No. 2, mayo-agosto, 2020

ISSN (impreso): 2613-8794 • ISSN (en línea): 2613-8808

DOI: https://doi.org/10.22206/cyed.2020.v4i2.pp27-37

\title{
Autoevaluación y acreditación universitaria en el contexto ecuatoriano. Perspectivas de análisis y mejora
}

\author{
Self-evaluation and university accreditation in the Ecuadorian \\ context: perspectives for analysis and improvement
}

\author{
Francisco Dillon ${ }^{a}$ ORCID: 0000-0002-8776-3435 \\ David Rojas ${ }^{b}$ ORCID: 0000-0003-2046-6636 \\ Jeanneth Robalino ${ }^{c}$ ORCID: 0000-0003-1559-1301 \\ Mirian Maldonado ${ }^{\mathrm{d}}$ ORCID: 0000-0001-9644-5677
}

Recibido: 29/01/2020 • Aprobado: 28/02/2020

Cómo citar: Dillon, F., Rojas, D., Robalino, J., \& Maldonado, M. (2020). Autoevaluación y acreditación universitaria en el contexto ecuatoriano. Perspectivas de análisis y mejora. Ciencia y Educación, 4(2), 27-37. Doi: https://doi.org/10.22206/cyed.2020.v4i2. pp27-37

\section{Resumen}

La investigación, vinculación y docencia, también denominados condiciones institucionales, son primordiales al momento de analizar las fortalezas y debilidades de una institución de educación superior que pretende realizar procesos de autoevaluación y acreditación. Sin un adecuado análisis de esta información, estos procesos pueden derivar en análisis equivocados y planteamientos erróneos de acciones con fines prospectivos y de mejora continua. El objetivo de este estudio fue determinar la influencia de la articulación de estos procesos como un ejercicio de autoevaluación con fines de acreditación; para esto, se utilizó como marco metodológico y de análisis la teoría de redes. Como conclusión se menciona que los procesos sustantivos universitarios, principalmente la docencia, influyen y fortalecen de manera determinante la comunicación académica y la producción científica diferenciada por género en docentes universitarios con fines de autoevaluación-acreditación.

Palabras clave: autoevaluación; docente; medios sociales; Ecuador.

\begin{abstract}
Research, bonding and teaching, also called institutional conditions, are essential when analyzing the strengths and weaknesses of a higher education institution that aims to carry out self-evaluation and accreditation processes. Without an adequate analysis of this information, these processes can lead to erroneous analyzes and erroneous approaches to actions with prospective and continuous improvement purposes. The objective of this study was to determine the influence of the articulation of these processes as a self-evaluation exercise for accreditation purposes; For this, network theory was used as a methodological and analytical framework. In conclusion, it is mentioned that the university substantive processes, mainly teaching, influence and strengthen academic communication and scientific production differentiated by gender in the university faculty for the purpose of self-evaluation-accreditation.
\end{abstract}

Keywords: self-appraisal; teacher; social media; Ecuador.

\footnotetext{
a Universidad Tecnológica Indoamérica. Quito-Ecuador. Correo-e: franciscodillon@uti.edu.ec

b Universidad Tecnológica Indoamérica. Quito-Ecuador. Correo-e: davidrojas@uti.edu.ec

c Unidad Educativa Fiscal “Nueva Aurora”. Quito-Ecuador. Correo-e: jeannethjr@hotmail.com

d Escuela de Educación Básica Estados Unidos de Norteamérica. Quito-Ecuador. Correo-e: maldonadomirian@hotmail.com
} 


\section{Introducción}

\section{La educación universitaria en el contexto ecuato- riano y su relación con los procesos de acreditación y autoevaluación institucional}

La Organización de los Estados Iberoamericanos para la Educación, la Ciencia y la Cultura (OEI) ha definido metas educativas con miras al año 2021; una de las más importantes (meta 21) se refiere al fortalecimiento de los sistemas educativos a través del proyecto "Metas Educativas - 2021", en cada uno de los países miembros de esta organización (Franco, Ortiz y Palacios; 2017). Uno de los ejes fundamentales de esta propuesta educativa, es la mejora continua de la educación superior y con esto, el adecuado desarrollo de competencias asociadas a los perfiles de egreso (salida-graduación-titulación) de cada una de las carreras y programas ofertadas en el contexto universitario y que, en última instancia, deberían responder a las demandas de conocimiento necesarias para el desarrollo de la sociedad. (Franco et al., 2017)

Bajo esta visión, el direccionamiento estratégico de la oferta académica que brindan las instituciones de educación superior (IES) en el Ecuador, en muchos casos, se encuentra alineado a la declaratoria de políticas educativas definidas por los organismos de control (Abad, López y Fernández; 2017). La pertinencia y calidad de esta oferta, además, cuenta con el sustento y base legal definidos para efecto en la Ley Orgánica de Educación Superior (LOES), su reglamento y el Reglamento de Régimen Académico (RRA). Estos principios, pertinencia y calidad, son evaluados de manera constante por instituciones rectoras de las políticas educativas a nivel superior (Consejo de Aseguramiento de la Calidad de la Educación Superior - CACES) a través de procesos de evaluación institucional con fines de acreditación (Abad et al., 2017; Ganga y Maluk, 2017; Portal y Keeling, 2017).

Por ejemplo, sobre lo mencionado, sería importante definir la tasa de titulación y la de eficiencia terminal de un programa o carrera, esto no solamente para medir cuantitativa o cualitativamente un indicador de gestión administrativa o académica universitaria, sino para medir la eficacia de los procesos de enseñanza-aprendizaje y los esfuerzos realizados desde la IES para desarrollar habilidades, destrezas y compe- tencias asociados a los perfiles de egreso de cada carrera o programa, permitiendo al estudiante universitario ser competitivo, competente, e insertarse de manera adecuada al ámbito profesional y laboral. (Armengol, Flores y Gairin, 2019; Dillon y Espinosa, 2018; Ganga et al., 2017; Portal et al., 2017)

El CACES, en el año 2019, evaluó algunas IES en función de parámetros conocidos como condiciones institucionales. Estas comprendían el análisis de los componentes de investigación, docencia y vinculación con la sociedad. La función sustantiva de docencia para su operatividad, se encuentra dividida en dos componentes: profesorado y estudiantado; las funciones sustantivas de investigación y vinculación con la sociedad, no tienen estos elementos; $y$, la función de apoyo condiciones institucionales, considera: planificación estratégica y operativa, infraestructura y equipamiento, bibliotecas, gestión interna de la calidad, bienestar estudiantil e igualdad de oportunidades.

Las funciones sustantivas se evalúan a la luz de tres dimensiones: planificación, ejecución y resultados. Este modelo de evaluación en el contexto ecuatoriano, plantea referentes de calidad que sirven para contrastar el cumplimiento de condiciones mínimas de calidad institucional, los cuales son: estándares fundamentales y proyectivos, elementos fundamentales, elementos proyectivos y fuentes de información (Universidad Tecnológica Indoamérica, 2019). Debido a esto, las IES, han definido procesos de autoevaluación institucional para medir en la práctica, cuáles de los parámetros establecidos en este modelo de evaluación se cumplen, y cuáles, a través de acciones de mejora y análisis prospectivos, se pueden cumplir con fines de acreditación y mejoramiento de la calidad de la educación ofertada. (Abad et al., 2017; Franco et al., 2017; Ponce et al., 2016; Universidad Tecnológica Indoamérica, 2019)

La autoevaluación institucional puede ser definida entonces como un proceso metodológico consciente de autorreflexión, que permite realizar una valoración cuali-cuantitativa de aquellas fortalezas y debilidades institucionales, con miras a la modificación o rectificación efectiva de las actividades y procesos que permitan mejorar la realidad del contexto analizado, en primera instancia. Este tipo de acciones de autorreflexión, parten del análisis independiente de cada una de las variables ejecutadas en esos contextos, en este caso en 
particular, el educativo. Por otro lado, los procesos de acreditación institucional, generalmente ejecutadas por organismos de control certificados y oficiales que dirigen el proceso académico y administrativo de las IES, parten también de un análisis cuali-cuantitativo de aquellas fortalezas y debilidades institucionales, esto, con la finalidad de adquirir un estatus o estado (acreditación) que permitirá, en última instancia, a la institución de educación superior (IES), continuar funcionando y proporcionando sus servicios académicos y de formación a la comunidad (Bolívar, 1994; Landi y Palacios, 2010; Murillo, 2008; Ruiz, 1995).

\section{Articulación de los procesos sustantivos universita- rios desde el análisis de redes sociales y académicas}

Sobre esta premisa, los ejes sustantivos sobre los cuales se realizan los procesos de autoevaluación y acreditación institucional en el contexto ecuatoriano, esto es, investigación, vinculación y docencia, también llamadas "condiciones institucionales", necesitan ser revisados bajo metodologías claras y definidas con anterioridad, para, de ser posible, analizar cada una de las variables que intervienen en su articulación y mejorarlas mediante acciones prospectivas. Los actores que participan en estas actividades docentes y administrativas dentro de una IES, lo hacen a través de relaciones sociales y académicas, utilizando por lo general el envío de información física o electrónica, mensajes de texto, conversaciones; entre otros. (Ahumada, Galdanes, Gonzales y Herrera, 2009; Granda y San Fabián, 2008; Santana y Quesada, 2008; Vigo, 2014)

Muchos de estos actores, por ende, tienden a relacionarse en función del eje sustantivo que rige sus actividades docentes o administrativas: investigación (a través de la escritura académica y científica), vinculación (a través de proyectos, planes o programas que coadyuvan a la interacción directa con la sociedad) o docencia (a través de la cátedra libre y flexible, respetando las condiciones curriculares de la institución); por esto, la teoría o análisis de redes sociales (ARS) proporciona al investigador las herramientas estadísticas necesarias para medir el grado de relación académica o administrativa y, a su vez, la articulación de estos ejes con fines de autoevaluación y acreditación.
(Aguilar, 2005; Albert y Barabási, 2002; Buela y Sierra, 2007; Bolívar et al., 1994; D Blondel, 2008)

En una red docente, por ejemplo, sería importante identificar aquellos nodos que se interrelacionan con otras carreras y cómo estas propician la escritura académica diferenciada por campo del conocimiento y por género (Dillon y Espinosa, 2020); además, se podría analizar, cómo las relaciones establecidas a través de un proyecto formativo (materia), permitieron mejorar los resultados de aprendizaje de sus estudiantes (Dillon y Espinosa, 2019); en el caso de la red de vinculación, sería interesante analizar, cómo la articulación de planes y programas de vinculación con la sociedad, han permitido a ese grupo atendido relacionarse y modificar aquella realidad del entorno que fue intervenida. Estos y otros análisis, como la capacidad instalada por ejemplo (Dillon, Espinosa et al., 2019), llevarían, considerablemente, a los procesos de autoevaluación y acreditación institucionales a otro tipo de estándares, que, para ser validados en el contexto ecuatoriano, deberían ser estudiadas en el tiempo. (Abramo, D’Angelo, y Caprasecca, 2008; Badar, Hite, y Badir, 2013; Estébanez, 2007; Ruiz, 1995)

Este estudio es un pequeño aporte que pretende analizar la influencia de la articulación de los ejes sustantivos universitarios (investigación, vinculación y docencia) en las relaciones docentes con fines de autoevaluación y acreditación, para de ser el caso, establecer acciones concretas de análisis y mejora continua para aquellos ejes que no tributen información valiosa con fines de autoevaluación y acreditación institucional. (Bolívar et al., 1994; Santana y Quesada et al., 2008; Vigo et al., 2014)

\section{Metodología}

La investigación buscó responder a las siguientes preguntas: ¿la topología de la red, influye en la articulación de los procesos sustantivos universitarios y en las relaciones docentes y de género? ¿Se evidencian de manera diferenciada (por género) la articulación de alguno de los procesos sustantivos universitarios?

El estudio se realizó en dos fases:

1. Construcción teórica: a través del análisis de las variables de estudio tomadas como referencia en este estudio: a) articulación de procesos 
sustantivos universitarios; y, b) las relaciones docentes a través del levantamiento de la topología de la red comunicacional docente;

2. Construcción del marco metodológico y análisis de resultados: a través del levantamiento de la información, su correlación de variables y el análisis estadístico y referencial de los resultados obtenidos. (Aguilar et al., 2005; Cestero et al., 2018).

El trabajo tuvo un enfoque cuantitativo, de corte transversal y alcance de tipo: exploratorio descriptivo y correlacional (Hernández, 2010). La población total de estudio estuvo compuesta por 459 individuos de una universidad particular, tomada del contexto ecuatoriano. En esta, se realizó un muestreo de tipo no probabilístico por conveniencia, de la cual se obtuvo una muestra de 373 individuos, que corresponden a la población tomada para el contexto de esta investigación (docentes universitarios que articulan los procesos sustantivos de investigación, vinculación y docencia).

Se realizó previa la aplicación del instrumento de investigación denominado: "RED_PROCESOS_SUS1", una validación de juicio de expertos (con la participación de tres (3) expertos en esta área de investigación) en el cual se determinó que el instrumento es válido y, complementariamente, se analizó el mismo en una muestra pequeńa (10 \% de la población de referencia) en donde se aplicó la medida Alfa Cronbach, cuyo resultado permitió determinar su nivel de confiabilidad en la aplicación: 0.984. (Cestero et al., 2018; George et al., 2003; Padua et al., 2018).

Posterior a la validación del instrumento, fue aplicado, utilizando una matriz de adyacencia (matriz de doble entrada con referencias de las relaciones docentes con "unos-1" y "ceros-0") para levantar la información de la articulación de los procesos sustantivos y la red comunicacional en los docentes universitarios. Los resultados obtenidos fueron ingresados a dos softwares especializados en el análisis de redes sociales (GEPHI y SOCNET) y un software estadístico (ORANGE versión 3.0.22); en donde se analizaron las medidas estadísticas de la red docente e influencia en la articulación de los procesos sustantivos universitarios diferenciados por género; los resultados obtenidos se presentan a continuación. (D Blondel et al., 2008; Park y Newman et al., 2004; Watts, 2006)

\section{Resultados}

\section{Red de interacciones académicas y de género}

Los docentes universitarios forman parte en la articulación de los procesos sustantivos universitarios, como agentes dinámicos; en la figura 1 se puede observar cómo estos se agrupan en función del tipo de relaciones académicas (clases, tipo de carrera y facultad, entre otras) que ejecutan y que tiene relación con la articulación de los procesos antes mencionados. En la figura 2 se puede observar los docentes que realizan actividades de investigación, en la figura 3, las actividades de vinculación, y en la figura 4, las actividades de docencia. Los nodos y su tamaño (puntos de color) representan a las personas que mejor articulan aquellos procesos sustantivos universitarios con fines de acreditación; el $11 \%$ de la totalidad docente, se dedica a realizar actividades de investigación; el $15 \%$ a actividades de vinculación, y el $79 \%$ a actividades de docencia. Sin embargo, se deben establecer algunas medidas estadísticas de la red para determinar la influencia de estos procesos sustantivos en las relaciones docentes y de género con fines de acreditación. (Albert y Barabási et al., 2002; Badar et al., 2013; Carayol y Matt, 2004; Fuchs, Stebut, y Allmendinger, 2001; Padua et al., 2018; Ponce et al., 2016)

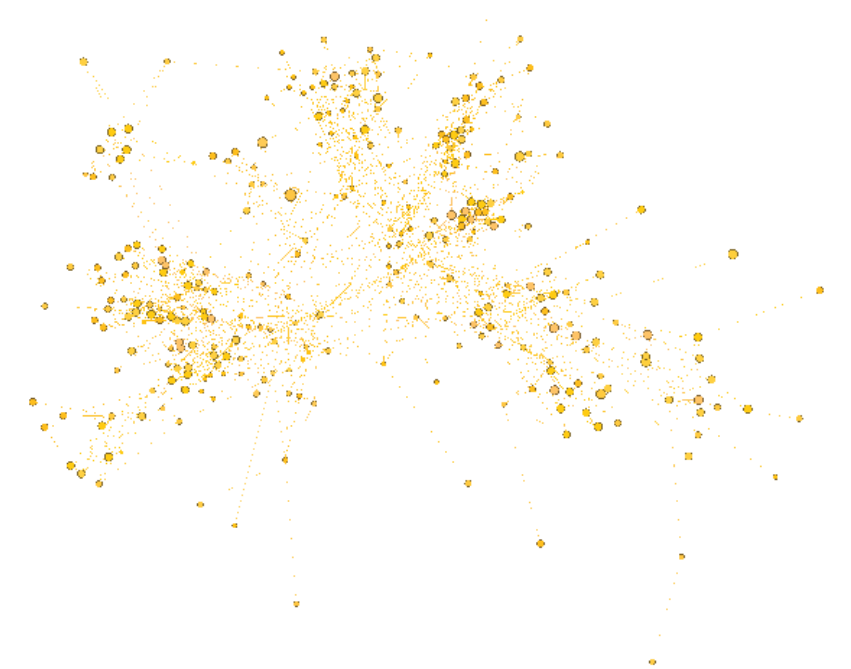

Figura 1. Articulación de los procesos sustantivos universitarios por parte de la población investigada

Fuente: elaboración propia. 


\section{Flujos de información, poder y estrés dentro de la red}

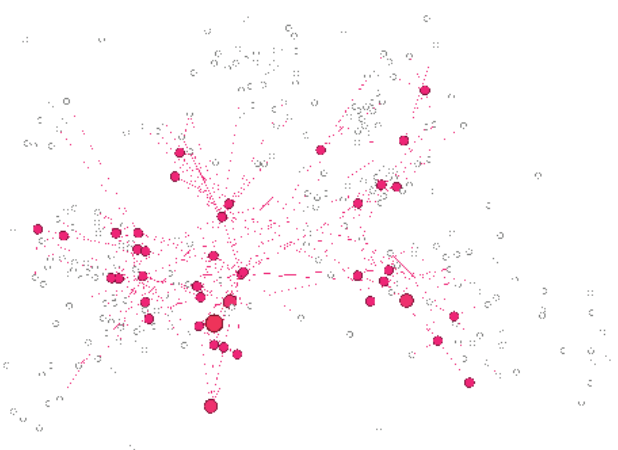

Figura 2. Red docente caracterizada por actividades de investigación

Fuente: elaboración propia.

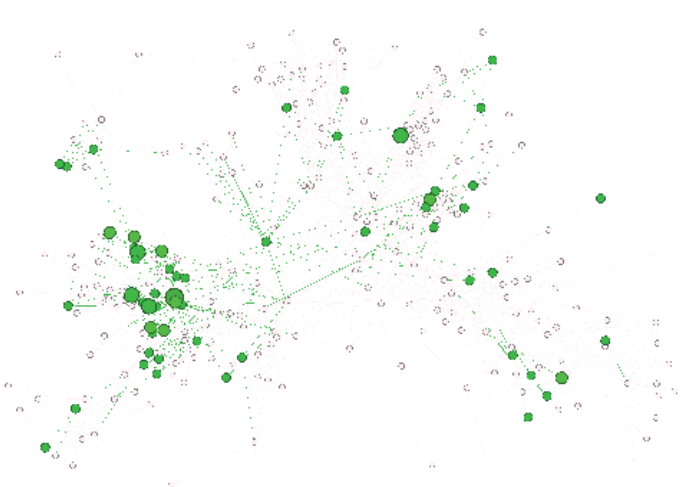

Figura 3. Red docente caracterizada por actividades de vinculación

Fuente: elaboración propia.

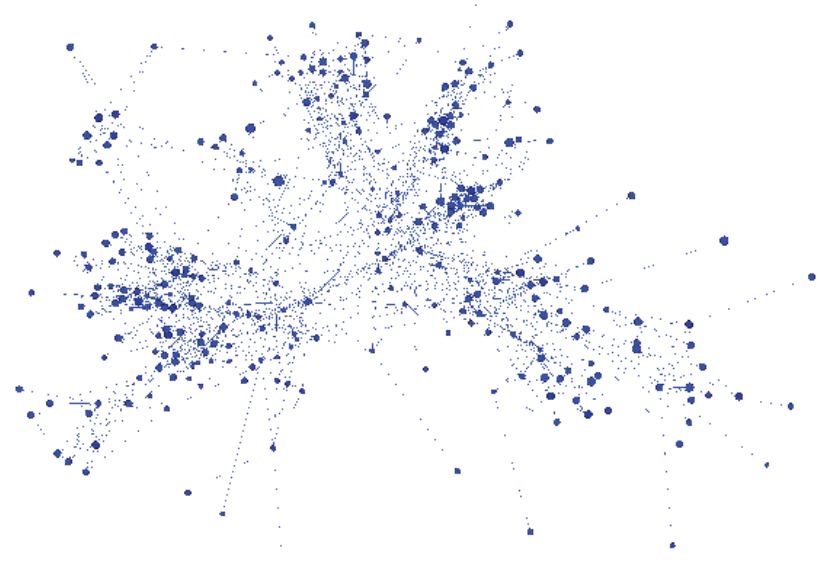

Figura 4. Red caracterizada por actividades de docencia (clases impartidas)

Fuente: elaboración propia.
Si observamos la caracterización de la topología de la red (ver figura 5) de docentes diferenciada por género, y utilizando el software SOCNET, se puede observar que los flujos de información dentro de la red (de color rojo los más importantes y de color azul los menos importantes), se encuentran dominados por los nodos masculinos (figuras cuadradas), mientras que los nodos femeninos (figuras circulares) se encuentran como intermediarias de los flujos de información; sin embargo, se debe destacar que existen tres nodos femeninos que tienen una presencia importante dentro del manejo de la información de la red. (Aguilar et al., 2005; Albert y Barabási et al., 2002)

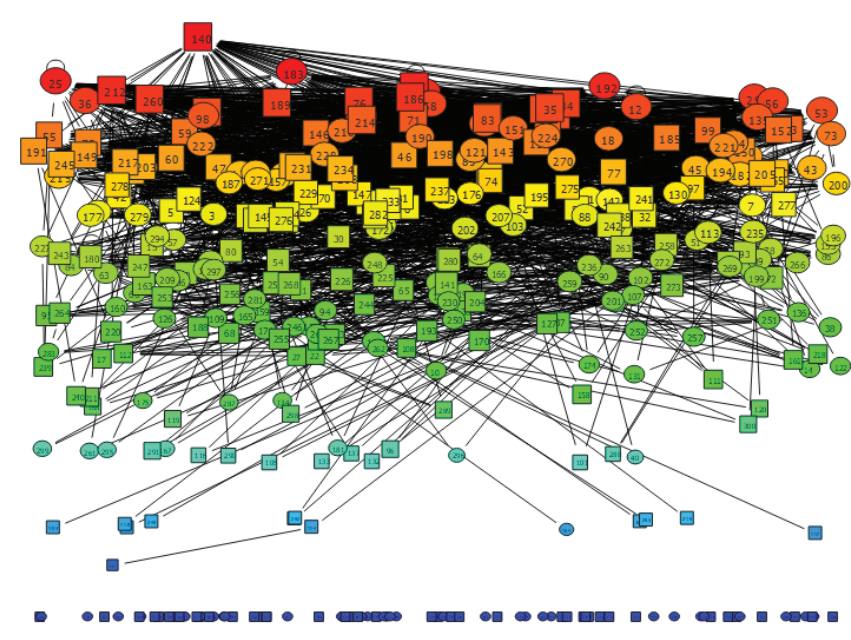

Figura 5. Red caracterizada en función del género y de los flujos de información que se transmiten

Fuente: elaboración propia.

Dentro de la caracterización de la topología de la red, también se pueden observar nodos que concentran el poder; entiéndase el mismo como el número de conexiones y relaciones que permiten a un nodo contar con una posición privilegiada dentro de la red al encontrarse más y mejor interconectado. En la figura 6, estos "flujos o conexiones de poder" (de color rojo los más importantes y de color azul los menos importantes), se encuentran dominados por un nodo femenino (figura circular), mientras que los nodos masculinos (figuras cuadradas), se encuentran como 
intermediarias de los flujos de poder dentro de la misma, estableciendo una relación inversa al análisis de la medida estadística anteriormente calculada. Esto podría deberse a la articulación de los ejes sustantivos universitarios o, a su vez, a la popularidad y grados de entrada de este nodo y otros en la red, lo que le permite en este caso contar con una posición privilegiada dentro de la misma. (Betz, 2010; Landi y Palacios 2010; Murillo et al., 2008; Naranjo, 2010)

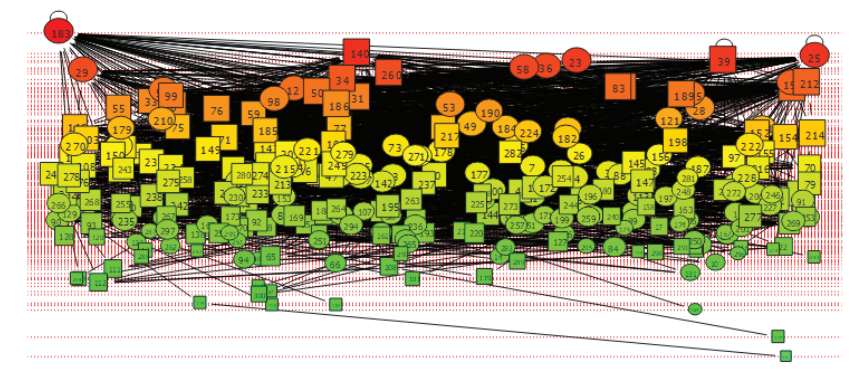

Figura 6. Red caracterizada en función del género y de los flujos de poder que se establecen entre los nodos de la red

Fuente: elaboración propia.

De la misma manera, si observamos la caracterización de la topología de la red (ver figura 7) en función del estrés de un nodo en la red (o la sobrecarga de interconexiones, funciones o procesos sustantivos en este caso) se puede observar, que muy pocos nodos femeninos (circulares) concentran poder y el flujo de información en la red (uno el más sobresaliente), mientras que los nodos masculinos (cuadrados) tienen una mayor demanda o sobrecarga en relación a sus funciones mismas que articulan los procesos de investigación, vinculación y docencia. El análisis de estas medidas estadísticas lo que evidencian es que los nodos masculinos controlan los flujos de información académica en la red, sin embargo, sin la interconexión mediada de los nodos femeninos, la información que en esta circula no fluiría de manera adecuada, lo que determina en este caso la importancia de los nodos femeninos en el manejo de los flujos comunicacionales mediales que permiten la interconexión de los otros nodos dentro de la red, justamente debido a esto, aquellos nodos que manejan los flujos comunicacionales y el poder, son los que mayor estrés evidencian dentro de la misma (ver figura 7) (Albert y Barabási, 2002; Alda y Fries, 2005; Requena, 1998)

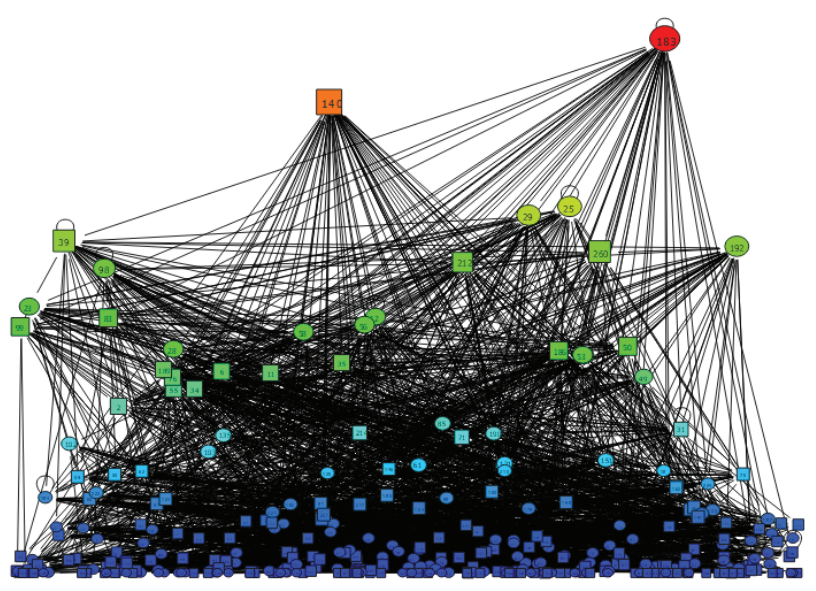

Figura 7. Red caracterizada en función del género y de los flujos de poder que se establecen entre los nodos de la red

Fuente: elaboración propia.

\section{Articulación de los ejes sustantivos en función de las relaciones docentes y de género}

Para responder a las preguntas planteadas, adicionalmente se ha realizado una correlación de las medidas estadísticas calculadas en la red a través de la utilización del software GEPHI (análisis de redes sociales) y ORANGE (para análisis estadísticos y correlacionales); los resultados son presentados a continuación:

- La función sustantiva docencia, no influye de manera directa en el número de conexiones que un nodo tanto masculino $(\mathrm{r}=-0.52)$ como femenino $(r=-0.50)$ mantiene en la red. (Ver figura 8) 


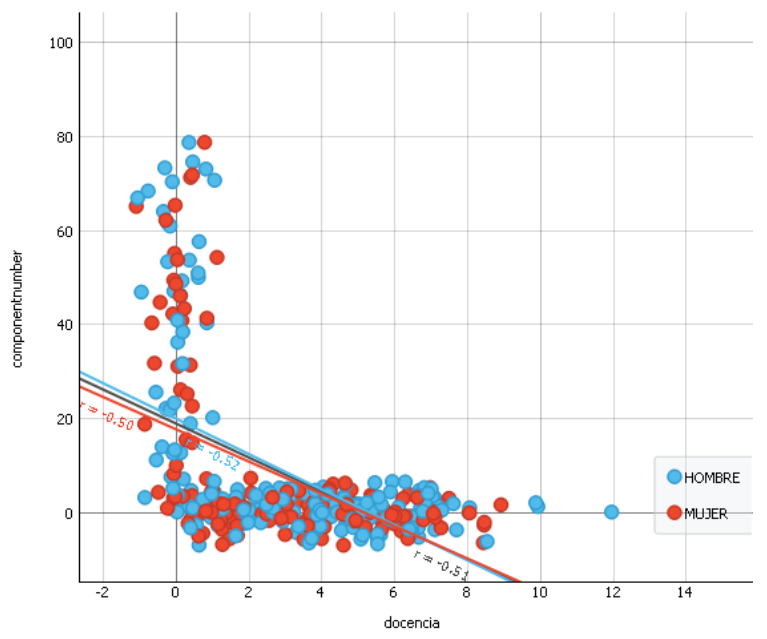

Figura 8. Correlación $\mathrm{N}^{\circ} 1$

Fuente: elaboración propia.

- El número de pasos que un nodo femenino $(r=0.56)$ en la red debe dar para conectarse con otros dentro de la red es más numeroso, esto debido a que son los hombres y no las mujeres los que manejan los flujos de comunicación dentro de la misma. (Ver figura 9)

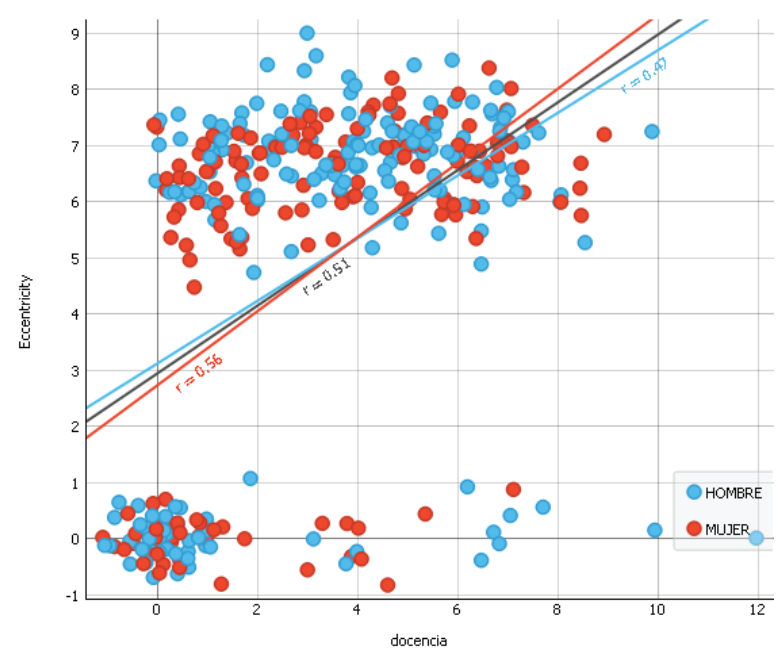

Figura 9. Correlación $\mathrm{N}^{\circ} 2$

Fuente: elaboración propia.

- La gestión docente no influye en la formación de grupos académicos ni mucho menos en el agrupamiento de docentes por área (facultad o carrera) tanto de los nodos masculinos $(\mathrm{r}=-0.53)$ como de los nodos femeninos $(r=-0.52)$, esto debido a que existe una fortaleza en la conformación de grupos docentes ya establecidos al momento de este estudio, mismos que permiten evidenciar en la docencia una fortaleza en la articulación de este eje sustantivo, indicador de acreditación (ver figura 10). De la misma manera se puede evidenciar que la gestión docente tampoco influye en la fuerza (fortaleza) de conexión que tienen los nodos masculinos $(r=-0.59)$ y femeninos $(r=-0.56)$ en la red. (Ver figura 11)

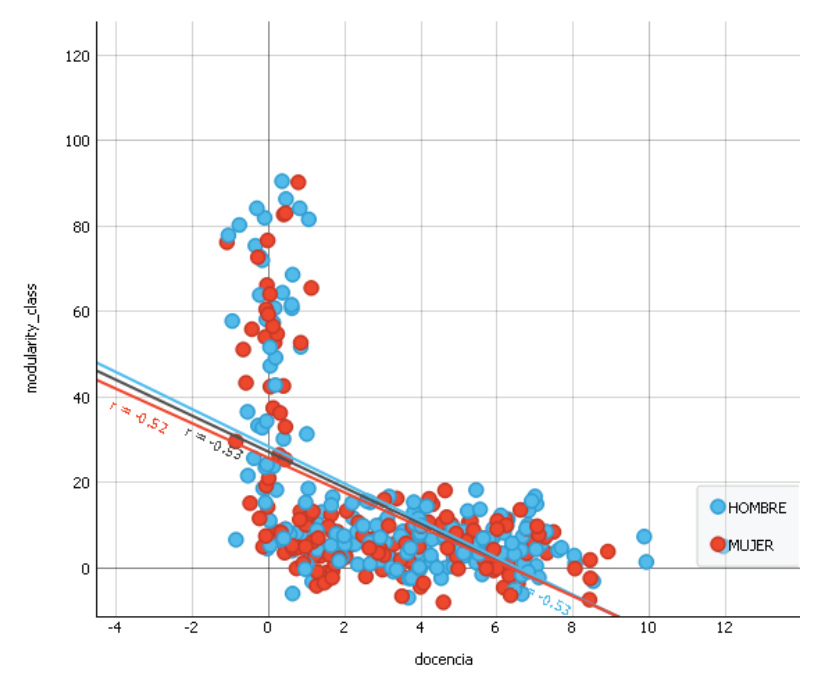

Figura 10. Correlación $\mathrm{N}^{\circ} 3$

Fuente: elaboración propia.

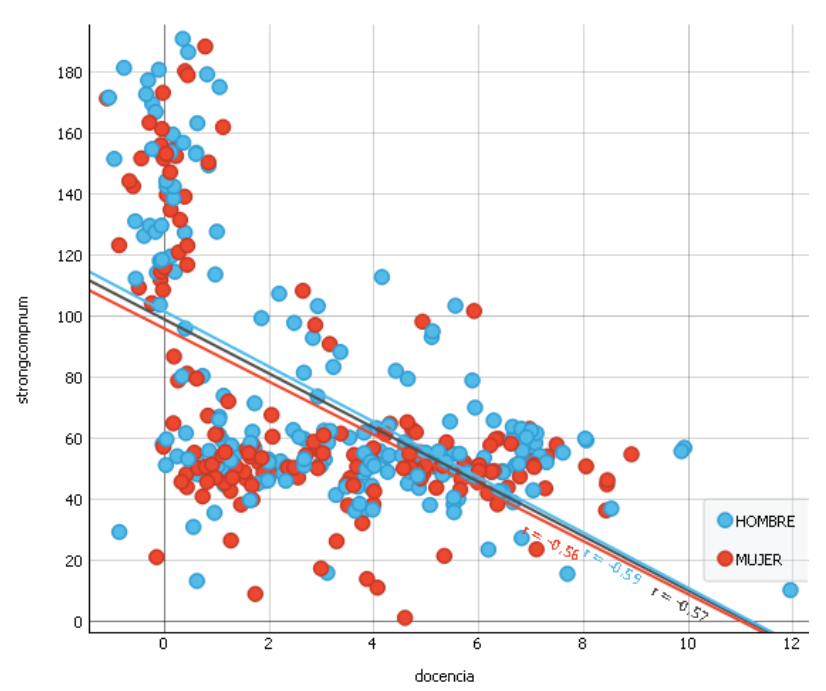

Figura 11. Correlación $\mathrm{N}^{\circ} 4$

Fuente: elaboración propia. 
- La función docencia tiene una relación estadística significativa de los nodos masculinos $(\mathrm{r}=0.53)$ y altamente significativa en los nodos femeninos $(\mathrm{r}=0.83)$ de la red en la articulación de la suma de los procesos sustantivos universitarios; esto quiere decir que las docentes (mujeres) son quienes mejor articulan este eje sustantivo "docencia", por ende, tienen más relevancia e importancia dentro de los análisis de los procesos docentes en la red. (Ver figura 12)

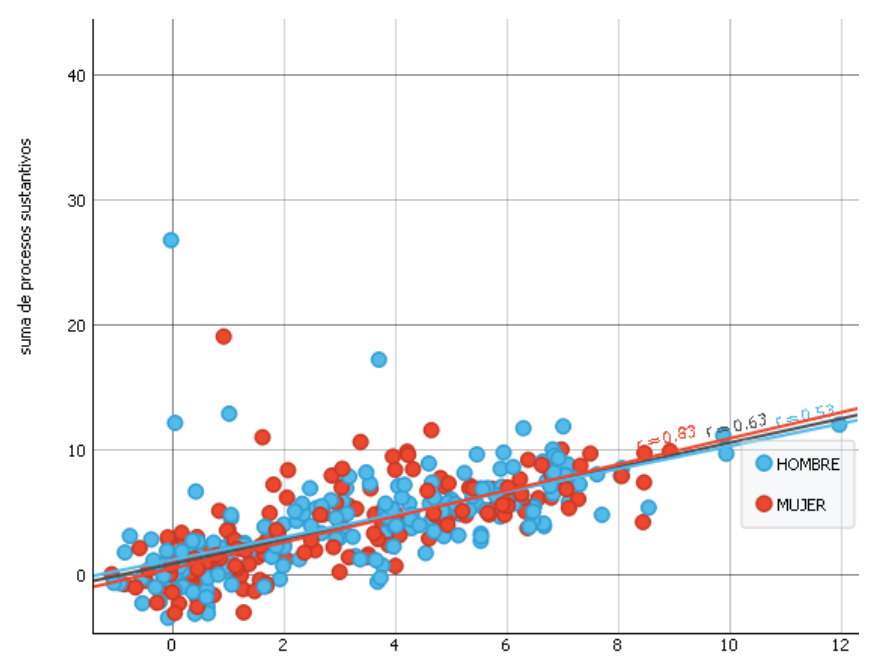

Figura 12. Correlación $N^{\circ} 5$

Fuente: elaboración propia.

- La función investigación manifiesta una relación estadísticamente significativa con la producción científica de los nodos femeninos en la red $(r=0.63)$; esto quiere decir que los procesos de producción científica (artículos en revistas indexadas, libros y capítulos de libros) de los nodos femeninos en la red, son los que más aportan a la articulación del eje sustantivo "investigación" y, por ende, a los procesos de autoevaluación y acreditación institucional (ver figura 13), esto se debe en gran medida a que la producción científica tiene como autoras y coautoras (principalmente) a un número significativo de docentes mujeres.

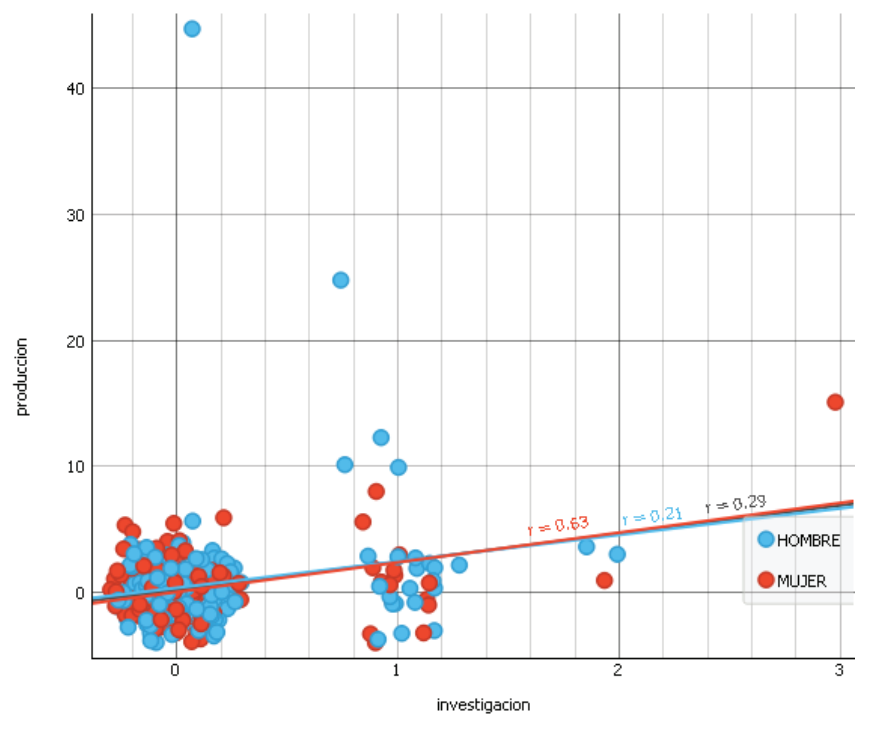

Figura 13. Correlación $\mathrm{N}^{\circ} 6$

Fuente: elaboración propia.

- Los procesos sustantivos de manera gene ral (investigación, vinculación y docencia), son mejor articulados por los nodos femeninos $(\mathrm{r}=0.57)$ dentro de la red. (Ver figura 14)

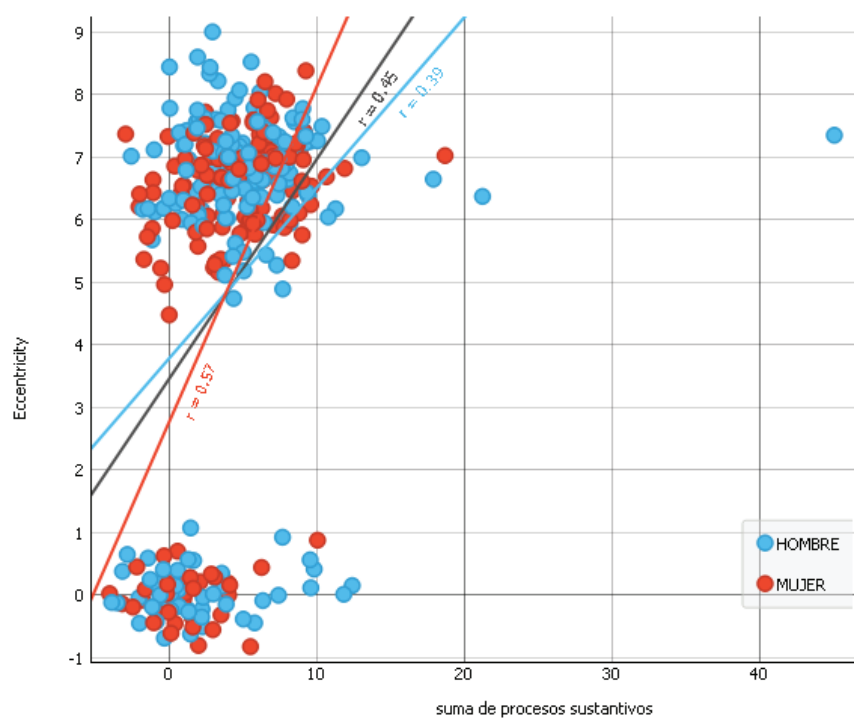

Figura 14. Correlación $\mathrm{N}^{\circ} 7$

Fuente: elaboración propia. 


\section{Conclusiones}

Los resultados obtenidos han permitido responder a las preguntas de investigación relacionadas con la influencia de la topología de la red docente en la articulación de los procesos sustantivos y, además, identificar la influencia de los flujos comunicacionales en las relaciones docentes que articulan estos procesos diferenciados por género; debido a esto se puede mencionar que:

La vinculación y docencia cumplen con acciones y actividades específicas que permiten identificar (ver figuras 2, 3 y 4) y medir estos los resultados con fines de autoevaluación y acreditación institucional. Para esto se tomó en cuenta solo algunos factores como: el número de materias dictado por docente, el número de artículos, libros y capítulos de libros escritos por docentes investigadores y el número de proyectos de vinculación ejecutados; mismos que permitieron caracterizar la topología de la red y poder estudiar las medidas estadísticas de esta. (Aguilar et al., 2005; Ahumada et al., 2009; Alda y Fries, 2005; Betz et al., 2010; Watts et al., 2006; Zippel et al., 2011)

La escritura académica por parte de docentes mujeres es la que, de manera directa, más tributa a la articulación del proceso sustantivo "investigación" en esta red, dejando claro que las estructuras y roles de poder, muchas veces diferenciados por género en la escritura de artículos científicos, no siempre y necesariamente aportan de manera significativa a este proceso con fines de acreditación. (Bolívar et al., 1994; Buela y Sierra et al. 2007; Cestero et al. 2018; D Blondel et al. 2008; Dillon y Espinosa et al. 2020; Estébanez et al. 2007; George et al. 2003; Gunnar et al. 2007; Rubido, Grebogi, y Baptista, 2017)

Además, la articulación de los procesos sustantivos universitarios en la estructura comunicacional docente diferenciada por género, tienen relación directa con la topología de la red investigada, de esto se desprende que hombres y mujeres forman parte de las estructuras de poder dentro de la red. Sin embargo, las mujeres, al contar con una posición intermediaria en las comunicaciones de la red, de alguna manera manejan los flujos de comunicación que por ella transitan y las ubican, en algunos casos, en posiciones privilegiadas que les permiten articular de mejor manera algunos ejes sustantivos dentro de la red (docencia y escritura de artículos científicos, por ejemplo). (Betz et al., 2010; Boschini y Sjogren, 2007; Naranjo et al., 2010; Newman y Reinert, 2016; Sonnert, 1995; Vigo et al., 2014)

Como recomendación, se sugiere que la presente investigación sea replicada en un futuro cercano, esto para verificar si las acciones prospectivas y de mejora continua, permitieron modificar la topología y estructura de la red en la articulación de los procesos sustantivos con fines de autoevaluación y acreditación.

\section{Referencias}

Abad G., López, M. L., y Fernández, K. L. (2017). El sistema de educación superior ecuatoriano visto desde los principios de pertinencia y calidad. Universidad y Sociedad, 9(5), 46-53.

Abramo, G., D’Angelo, C., y Caprasecca, A. (2008). Gender differences in research productivity: A bibliometricanalysis of the Italian academic system. Scientometrics, 79(3), 517-539.

Aguilar, N. (2005). Manual introductorio al análisis de redes sociales. Revista Redes, 29(1), 16-43.

Albert , R., y Barabási, A. (2002). Statistical mechanics of complex networks. Reviews of modern physics, 74(1), 47-97.

Alda, F., y Fries, L. (2005). Feminismo, género y patriarcado. Revista sobre enseñanza del derecho en Buenos Aires, 3(6) 259-294.

Ahumada, L., Galdames, S., González, A., y Herrera, P. (2009). El funcionamiento del equipo directivo durante un proceso de autoevaluación institucional en el marco de políticas de aseguramiento de la calidad de la gestión escolar en Chile. Universitas Psychologica, 8(2), 353-370. Recuperado de https://revistas.javeriana.edu.co/ index.php/revPsycho/article/view/264

Armengol, C., Flores, Q. P., y Gairín, J. (2019). Acreditación, educación superior y respuesta del profesorado entre 2008-2017. Revista de Educación Superior del Sur Global - RESUR, 7(1), 21-37. 
Badar, K., Hite, J., y Badir, Y. (2013). Examining the relationship of co-authorship network centrality and gender on academic research performance: The case of chemistry researchers in Pakistan. Scientometrics, 94(2), 755-775.

Betz, B. (2010). Análisis de las fortalezas institucionales para la contratación de invest igadores. Aula Abierta, 38(2), 65-74.

Bolívar, A. (1994). Autoevaluación institucional para la mejora interna. Reforma educativa y organización escolar, 1(1), 915-944.

Boschini, A., y Sjogren, A. (2007). Is team formation gender neutral? Evidence from coauthorship patterns. Journal of Labor Economics, 25(2) 325-365.

Buela, G., y Sierra, J. (2007). Criterios, indicadores y estándares para la acreditación de profesores titulares y catedráticos de universidad. Psicothema 19(4), 537-551.

Carayol, N., y Matt, M. (2004). Does research organization influence academic production? Research Policy, 33(8), 1081-1102.

Cestero, E. (2018). Data Science y Redes Complejas Métodos y Aplicaciones. Madrid: Editorial Universitaria Ramon Areces.

D Blondel, V. (2008). Fast unfolding of communities in large networks. Journal of Statistical Mechanics: Theory and Experiment 1(1), 1-6.

Dillon, F., y Espinosa, D. (2020). Relaciones académicas y de género en docentes universitarios y su influencia en la publicación en revistas indexadas de alto impacto. Ciencia Y Educación, 3(3), 7-20.

Dillon, F., y Espinosa, D. (2019). Innovación y liderazgo educativo y su influencia en las relaciones sociales y académicas de estudiantes universitarios de posgrado. SATHIRI, 14(2), 138 - 150.

Dillon, F., Espinosa, D., Dillon, J. E., y Espinosa, P. (2019). Capacidad instalada, un factor para la optimización del tiempo y los recursos en instituciones de educación superior. Wimblu, 14(2), 47-57.

Dillon, F., y Espinosa, D. (2018). Empleabilidad en el Ecuador desde una perspectiva de género. Cienciamérica, 7(2), 98-112.
Estébanez, M. (2007). Género e investigación científica en las universidades latinoamericanas. Nueva Época, 1(12) 3-26.

Franco, J., Ortiz, L., y Palacios, Z. (2017). Formación del profesorado universitario hacia el año 2020: modelos educativos de vanguardia para la docencia agropecuaria de alta competencia en el Ecuador. Alternativas, 17(2), 11-34.

Fuchs, S., Stebut, J., y Allmendinger, J. (2001). Gender, science, and scientific organizations in Germany. Minerva, 39(1) 175-201.

Ganga, F. A. y Maluk, S. A. (2017). Análisis descriptivo del gobierno universitario ecuatoriano: una mirada desde los cambios legislativos. Revista Electrónica de Investigación Educativa, 19(2), 22-37.

George, D. (2003). Spss for Windows step by step: A Simple Guide and Reference. 11.0 Update. Boston: Allyn y Bacon.

Granda, A. y San Fabián J. (2008). Fases en la autoevaluación de un centro. Revista del Fórum Europeo de Administradores de la Educación, 16(1), 1001-1012.

Gunnar, H. (2007). Gender indicators in science, engineering and technology. An Information Toolkit. Paris: UNESCO.

Hernández, R. (2010). Metodología de la Investigación. México D.F.: Interamericana Editores S.A.

Landi, N. y Palacios, M. (2010). La autoevaluación institucional y la cultura de la participación; Revista Iberoamericana de Educación; 53(1), 155-181.

Murillo, P. (2008). La autoevaluación institucionalun camino importante para la mejora de los centros educativos. Revista del Fórum Europeo de Administradores de la Educación, 16(1), 13-17.

Naranjo, A. (2010). Estudio del efecto de la topología de mundo pequeño en el modelo de Ising de $1 \mathrm{D}$ y $2 D$, mediante simulación computacional, (tesis de grado). Universidad Politécnica Nacional. Quito, Ecuador.

Organización de Estados Iberoamericanos. (2010). Metas Educativas 2021: la educación que queremos para la generación de los Bicentenarios. Organización de Estados Iberoamericanos para la Educación, la Ciencia y la Cultura. ISBN: 978-84-7666-224-3. Recuperado de: http:// www.oei.es/metas2021/libro.htm. 
Padua, J. (2018). Técnicas de Investigación aplicadas a las ciencias sociales. Ciudad de México: Fondo de Cultura Económica.

Park, J., y Newman, M. (2004). Statistical mechanics of networks. Physical Review, 70(6), 1-15.

Ponce, J. (2016). Educación Superior en Iberoamérica - Informe 2016. Quito, Ecuador: CINDA - Informe Nacional Ecuador.

Portal, J., Pérez, L., y Keeling, M. (2017). La autoevaluación como parte del proceso de evaluación y acreditación institucional. VARONA, 65(1), $1-9$.

Requena, F. (1998). Género, redes de amistad y rendimiento académico. Papers, 56(1), 233-242.

Rubido, N., Grebogi, C., y Baptista, M. (2017). Understanding Information Transmission in complex networks, 387(1), 1-5.

Ruiz, J. M. (1995). La autoevaluación institucional en un centro de educación primaria. Revista Iberoamericana de Educación, 8(1), 1-23.

Santana, P. y Quesada, C. (2008). De la revisión interna a los planes de mejoratrazando una ruta. Revista del Fórum Europeo de Administradores de la Educación, 16(1), 24-28.
Sonnert, G. (1995). Gender differences in science careers. New Brunswick: Rutgers University Press.

Universidad Tecnológica Indoamérica, (2019). El aseguramiento de la calidad y la interacción de las funciones sustantivas. Quito: Universidad Tecnológica Indoamérica.

Vigo, P., Segrea González, J., León Sánchez, B., López Otero, T., Pons Mena, J. y León Sánchez, C. (2014). Institutional Self-assessment. An Essential Tool for Quality of University Processes. MediSur, 12(5), 727-735. Recuperado de: http://scielo.sld.cu/scielo.php?script=sci_arttext\&pid=S1727-897X2014000500008\&ln$\mathrm{g}=\mathrm{es} \& \mathrm{t} \operatorname{lng}=\mathrm{en}$.

Watts, D. (2006). A seis grados de separación. Barcelona: Paidós Ibérica, S.A.

Zippel, K. (2011). How gender neutral are state policies on science and international mobility of academics? Sociologica, 1(1), 1-19. 